\title{
RELAXATION APPROXIMATION OF SOME NONLINEAR MAXWELL INITIAL-BOUNDARY VALUE PROBLEM*
}

\author{
GILLES CARBOU ${ }^{\dagger}$ AND BERNARD HANOUZET ${ }^{\ddagger}$
}

\begin{abstract}
Two nonlinear Maxwell systems are considered: Kerr model exhibiting an instantaneous response of the medium, Kerr-Debye model which contains some delay term and is a relaxation approximation of the first one. In one space dimension, we prove that the limit of the solution to the ingoing wave condition for Kerr-Debye model is a solution to the Kerr model.
\end{abstract}

Key words. Initial boundary value problem, Kerr model, Kerr-Debye model, relaxation, nonlinear Maxwell equation.

AMS subject classifications. 35L50, 35Q60.

\section{Introduction}

Nonlinear Maxwell's equations are used for modelling nonlinear optical phenomena. The wave propagation in an isotropic medium is described by Maxwell's system:

$$
\begin{aligned}
& \partial_{t} D-\operatorname{curl} H=0, \\
& \partial_{t} B+\operatorname{curl} E=0, \\
& \operatorname{div} D=\operatorname{div} B=0 .
\end{aligned}
$$

The field quantities $E$ and $H$ represent the electric and magnetic fields, $D$ and $B$ the electric and magnetic displacements. We consider the constitutive relations for a nonlinear Kerr medium:

$$
\begin{aligned}
& B=\mu_{0} H, \\
& D=\varepsilon_{0} E+P,
\end{aligned}
$$

where $P$ is the nonlinear polarization.

If the medium exhibits an instantaneous response we have a Kerr model:

$$
P=P_{K}=\varepsilon_{0} \varepsilon_{r}|E|^{2} E .
$$

If the medium exhibits a finite response time $\tau$ we have a Kerr-Debye model:

$$
P=P_{K D}=\varepsilon_{0} \chi E,
$$

where

$$
\partial_{t} \chi+\frac{1}{\tau} \chi=\frac{1}{\tau} \varepsilon_{r}|E|^{2}
$$

(see for example [15] or [20]).

So the Kerr-Debye model is a relaxation approximation of the Kerr model and $\tau$ is the relaxation parameter (for a general presentation of relaxation problems, see [13]). Formally, when $\tau$ tends to $0, \chi$ converges to $\varepsilon_{r}|E|^{2}$ and $P_{K D}$ converges to $P_{K}$.

${ }^{*}$ Received: January 31, 2006; accepted (in revised version): March 7, 2006. Communicated by Lorenzo Pareschi.

†Mathématiques Appliquées de Bordeaux, UMR 5466 et Université Bordeaux 1, 351 cours de la Libération, 33405 Talence cedex, France.

¥Mathématiques Appliquées de Bordeaux, UMR 5466 et Université Bordeaux 1, 351 cours de la Libération, 33405 Talence cedex, France. 
Recently for the Cauchy problem with regular initial data, the convergence result was obtained in [7] and [8] using the general framework given in [17]. This study is based on a precise description of the boundary layer in time.

For the modelling of realistic physical situations it is more convenient to take into account boundary value conditions. In particular we consider the impedance boundary value problem. For the Kerr-Debye model in two space dimension, numerical studies were proposed for the Dirichlet condition on the magnetic field in [19] and for the ingoing boundary condition in [8].

The aim of this paper is to prove convergence results in the case of a impedance initial-boundary value problem for the Kerr-Debye model in one space dimension.

\section{The one space dimension models}

Let us suppose that

$$
\begin{aligned}
& D(x, y, z)=(0, d(x), 0) \\
& H(x, y, z)=(0,0, h(x))
\end{aligned}
$$

Then Maxwell's system can be rewritten as

$$
\begin{aligned}
& \partial_{t} d+\partial_{x} h=0, \\
& \partial_{t} h+\partial_{x} e=0,
\end{aligned}
$$

for $(t, x) \in([0,+\infty])^{2}$.

Once nondimensionalized the Kerr model, denoted by $(\mathrm{K})$, becomes:

$$
\left\{\begin{array}{l}
\partial_{t} d+\partial_{x} h=0, \\
\partial_{t} h+\partial_{x} e=0, \\
d=\left(1+e^{2}\right) e,
\end{array}\right.
$$

for $(t, x) \in\left(\mathbb{R}^{+}\right)^{2}$.

We suppose that the initial data vanishes

$$
d(0, x)=h(0, x)=0 \text { for } x \in \mathbb{R}^{+},
$$

and that we have the boundary condition

$$
h(t, 0)+a e(t, 0)=g(t) \text { for } t \in \mathbb{R}^{+},
$$

where $a$ is a non negative constant.

The system $(\mathrm{K})$ is quasi-linear hyperbolic. It is a p-system where $p$ is the reciproque function of $e \mapsto\left(1+e^{2}\right) e$, and it is strictly hyperbolic with eigenvalues

$$
\lambda_{1}=-\sqrt{p^{\prime}(d)}<0<\lambda_{2}=\sqrt{p^{\prime}(d)} .
$$

The energy density $\mathcal{E}_{K}$ given by

$$
\mathcal{E}_{K}(e, h)=\frac{1}{2}\left(e^{2}+h^{2}+\frac{3}{2} e^{4}\right)
$$


is a strictly convex entropy. In the entropic variable $(e, h)$, system $(\mathrm{K})$ writes in the symmetric form:

$$
\left(\begin{array}{cc}
1+3 e^{2} & 0 \\
0 & 1
\end{array}\right) \partial_{t}\left(\begin{array}{l}
e \\
h
\end{array}\right)+\left(\begin{array}{ll}
0 & 1 \\
1 & 0
\end{array}\right) \partial_{x}\left(\begin{array}{l}
e \\
h
\end{array}\right)=0
$$

The boundary $\{x=0\}$ is non characteristic. We can verify that the boundary condition (2.3) is an ingoing wave condition and is maximal dissipative (since $a \geq 0$ ). Therefore classical existence results of a regular solution for the $(\mathrm{K})$ boundary value problem (2.1)-(2.2)-(2.3) apply.

The one dimensional Kerr-Debye model, denoted by (KD) system, writes:

$$
\left\{\begin{array}{l}
\partial_{t} d_{\varepsilon}+\partial_{x} h_{\varepsilon}=0 \\
\partial_{t} h_{\varepsilon}+\partial_{x} e_{\varepsilon}=0 \\
\partial_{t} \chi_{\varepsilon}=\frac{1}{\varepsilon}\left(e_{\varepsilon}^{2}-\chi_{\varepsilon}\right) \\
d_{\varepsilon}=\left(1+\chi_{\varepsilon}\right) e_{\varepsilon}
\end{array}\right.
$$

for $(t, x) \in\left(\mathbb{R}^{+}\right)^{2}$, with initial data

$$
d_{\varepsilon}(0, x)=h_{\varepsilon}(0, x)=\chi_{\varepsilon}(0, x)=0 \text { for } x \in \mathbb{R}^{+}
$$

and with boundary condition

$$
h_{\varepsilon}(t, 0)+a e_{\varepsilon}(t, 0)=g(t) \text { for } t \in \mathbb{R}^{+} .
$$

Using the third equation in (2.6) and (2.7) we remark that

$$
\chi_{\varepsilon}(t, x) \geq 0 .
$$

The system (KD) is quasi-linear strictly hyperbolic with eigenvalues

$$
\lambda_{1}=\left(1+\chi_{\varepsilon}\right)^{-\frac{1}{2}}<\lambda_{2}=0<\lambda_{3}=\left(1+\chi_{\varepsilon}\right)^{-\frac{1}{2}} .
$$

The energy density $\mathcal{E}_{K D}$ given by

$$
\mathcal{E}_{K D}(d, h, \chi)=\frac{1}{2}(1+\chi)^{-1} d^{2}+\frac{1}{2} h^{2}+\frac{1}{4} \chi^{2}
$$

is a strictly convex entropy in $\{\chi \geq 0\}$, so the system (KD) is symmetrizable.

Using the entropy variables given by

$$
\left\{\begin{array}{l}
\partial_{d} \mathcal{E}_{K D}(d, h, \chi)=(1+\chi)^{-1} d:=e \\
\partial_{h} \mathcal{E}_{K D}(d, h, \chi)=h \\
\partial_{\chi} \mathcal{E}_{K D}(d, h, \chi)=-\frac{1}{2}(1+\chi)^{-2} d^{2}+\frac{1}{2} \chi=\frac{1}{2}\left(\chi-e^{2}\right):=v
\end{array}\right.
$$


system (KD) takes the symmetric form

$$
\left\{\begin{array}{l}
\left(1+3 e_{\varepsilon}^{2}+2 v_{\varepsilon}\right) \partial_{t} e_{\varepsilon}+2 e_{\varepsilon} \partial_{t} v_{\varepsilon}+\partial_{x} h_{\varepsilon}=0 \\
\partial_{t} h_{\varepsilon}+\partial_{x} e_{\varepsilon}=0 \\
2 e_{\varepsilon} \partial_{t} e_{\varepsilon}+2 \partial_{t} v_{\varepsilon}=-\frac{2}{\varepsilon} v_{\varepsilon} .
\end{array}\right.
$$

If we use the following notations:

$$
\begin{aligned}
& W_{\varepsilon}=\left(U_{\varepsilon}, v_{\varepsilon}\right) \text { with } U_{\varepsilon}=\left(e_{\varepsilon}, h_{\varepsilon}\right), \\
& A_{0}\left(W_{\varepsilon}\right)=\left(\begin{array}{ccc}
1+3 e_{\varepsilon}^{2}+2 v_{\varepsilon} & 0 & 2 e_{\varepsilon} \\
0 & 1 & 0 \\
2 e_{\varepsilon} & 0 & 2
\end{array}\right), A_{1}=\left(\begin{array}{lll}
0 & 1 & 0 \\
1 & 0 & 0 \\
0 & 0 & 0
\end{array}\right), \\
& Q\left(W_{\varepsilon}\right)=\left(\begin{array}{c}
0 \\
0 \\
v_{\varepsilon}
\end{array}\right)
\end{aligned}
$$

we obtain

$$
A_{0}\left(W_{\varepsilon}\right) \partial_{t} W_{\varepsilon}+A_{1} \partial_{x} W_{\varepsilon}=-\frac{2}{\varepsilon} Q\left(W_{\varepsilon}\right) .
$$

The matrix $A_{0}\left(W_{\varepsilon}\right)$ is definite positive because $\chi_{\varepsilon}=e_{\varepsilon}^{2}+2 v_{\varepsilon} \geq 0$ by (2.9). The matrix $A_{1}$ is singular with a one dimensional kernel, so the boundary $\{x=0\}$ is characteristic of constant multiplicity. In addition, the boundary condition (2.8) is an ingoing wave boundary condition and is maximally dissipative. Therefore the general results of [5] apply to obtain an existence result of regular solutions for the (KD) boundary value problem (2.6)-(2.8).

Now let us specify the assumptions on the source term $g$ in the boundary condition (2.3) or (2.8). For the modelling of realistic physical situations we can assume that $g$ is compactly supported in $[0,+\infty]$. We denote by $H^{s}$ the classical Sobolev space and we suppose that $g$ belongs to $H^{s}(\mathbb{R})$ for $s$ great enough. So the boundary condition and the null initial data match each other and we obtain smooth solutions.

In order to obtain a homogeneous boundary condition we replace the magnetic field $h$ with $h(t, x)+g(t) \eta(x)$ where $\eta$ is a smooth function, compactly supported in $\mathbb{R}^{+}$, equal to 1 in the neighborhood of 0 . We rewrite the $(\mathrm{K})$ boundary value problem in the entropic variables

$$
\left\{\begin{array}{l}
\left(\begin{array}{cc}
1+3 e^{2} & 0 \\
0 & 1
\end{array}\right) \partial_{t}\left(\begin{array}{l}
e \\
h
\end{array}\right)+\left(\begin{array}{ll}
0 & 1 \\
1 & 0
\end{array}\right) \partial_{x}\left(\begin{array}{l}
e \\
h
\end{array}\right)=\left(\begin{array}{l}
g_{1} \\
g_{2}
\end{array}\right) \text { for }(t, x) \in\left(\mathbb{R}^{+}\right)^{2}, \\
e(0, x)=h(0, x)=0 \text { for } x \in \mathbb{R}^{+}, \\
h(t, 0)+a e(t, 0)=0 \text { for } t \in \mathbb{R}^{+},
\end{array}\right.
$$

where $g_{1}(t, x)=-g(t) \eta^{\prime}(x)$ and $g_{2}(t, x)=-g^{\prime}(t) \eta(x)$, and we consider a regular solution of this problem. 
Proposition 2.1. There exists $T^{*}>0$ and a unique regular solution $U=(e, h)$ for the $(K)$ boundary value problem (2.11) defined in $\left[0, T^{*}\right] \times \mathbb{R}^{+}$and such that

$$
\partial_{t}^{i} U \in \mathcal{C}^{0}\left(\left[0, T^{*}\right] ; H^{2-i}\left(\mathbb{R}^{+}\right)\right) \text {for } i=0,1,2 .
$$

In the same way we rewrite the $(\mathrm{KD})$ boundary value problem with the entropic variables

$$
\left\{\begin{array}{l}
A_{0}\left(W_{\varepsilon}\right) \partial_{t} W_{\varepsilon}+A_{1} \partial_{x} W_{\varepsilon}=-\frac{2}{\varepsilon} Q\left(W_{\varepsilon}\right)+G(t, x) \text { for }(t, x) \in\left(\mathbb{R}^{+}\right)^{2}, \\
W_{\varepsilon}(0, x)=0 \text { for } x \in \mathbb{R}^{+}, \\
h_{\varepsilon}(t, 0)+a e_{\varepsilon}(t, 0)=0 \text { for } t \in \mathbb{R}^{+},
\end{array}\right.
$$

where $G={ }^{t}\left(g_{1}, g_{2}, 0\right)$.

For fixed $\varepsilon>0$, using the results in [5] we consider a regular local solution of this problem.

Proposition 2.2. There exists $T_{\varepsilon}^{*}>0$ and a unique regular solution $W_{\varepsilon}=\left(U_{\varepsilon}, v_{\varepsilon}\right)$ for the (KD) boundary value problem (2.12) defined on $\left[0, T_{\varepsilon}^{*}\right] \times \mathbb{R}^{+}$and such that

$$
\partial_{t}^{i} W_{\varepsilon} \in \mathcal{C}^{0}\left(\left[0, T_{\varepsilon}^{*}\right] ; H^{2-i}\left(\mathbb{R}^{+}\right)\right), i=0,1,2 .
$$

The goal of this paper is to analyse the behaviour of the regular solutions for the (KD) boundary value problem when the relaxation parameter $\varepsilon$ tends to 0 . By energy estimates we first obtain a uniform bound of $W_{\varepsilon}$ in the following theorem.

Theorem 2.3. Let $W_{\varepsilon}$ and $T_{\varepsilon}^{*}$ be given by Proposition 2.2. There exist $\tilde{T}>0$ and a constant $K>0$ such that for all $\varepsilon>0, T_{\varepsilon}^{*} \geq \tilde{T}$ and the solution $W_{\varepsilon}=\left(U_{\varepsilon}, v_{\varepsilon}\right)$ of the (KD) boundary value problem (2.12) satisfies

$$
\left\{\begin{array}{l}
\left\|\partial_{t}^{i} U_{\varepsilon}\right\|_{\mathcal{C}^{0}\left([0, \tilde{T}] ; H^{2-i}\left(\mathbb{R}^{+}\right)\right)} \leq K \text { for } i=0,1,2, \\
\frac{1}{\varepsilon}\left\|v_{\varepsilon}\right\|_{\mathcal{C}^{0}\left([0, \tilde{T}] ; H^{1}\left(\mathbb{R}^{+}\right)\right)} \leq K \\
\left\|\partial_{t}^{i} v_{\varepsilon}\right\|_{\mathcal{C}^{0}\left([0, \tilde{T}] ; H^{2-i}\left(\mathbb{R}^{+}\right)\right)} \leq K \text { for } i=0,1,2 .
\end{array}\right.
$$

Theorem 2.3 shows the strong convergence of $v_{\varepsilon}=\frac{1}{2}\left(\chi_{\varepsilon}-e_{\varepsilon}^{2}\right)$ to zero. The convergence of $U_{\varepsilon}$ to the solution of the boundary value problem (2.11) for (K) is contained in the following statement.

Theorem 2.4. Let $U$ and $T^{*}$ be given by Proposition 2.1, let $U_{\varepsilon}$ and $\tilde{T}$ be given by Theorem 2.3. For $T \leq \tilde{T}$ and $T<T^{*}$, there exists a constant $K>0$ such that for all $\varepsilon>0$,

$$
\left\|U_{\varepsilon}-U\right\|_{\mathcal{C}^{0}\left([0, T] ; L^{2}\left(\mathbb{R}^{+}\right)\right)} \leq K \varepsilon
$$


So we have proven that the regular solutions for the (KD) boundary value problem tend to the solution for the $(\mathrm{K})$ boundary value problem when the relaxation parameter tends to zero.

In our study we remark that no boundary layer appears in the time variable because the null initial data belongs to the equilibrium manifold defined by

$$
\mathcal{V}=\left\{(d, h, \chi) \text { such that } \chi-(1+\chi)^{-2} d^{2}=2 v=0\right\} .
$$

For the space variable, we have the same boundary condition for the system $(\mathrm{K})$ and for the system (KD), so no space boundary layer appears again.

In the case of non characteristic boundary conditions, a general study of boundary conditions for hyperbolic relaxation systems is given in [16].

To our knowledge general convergence results are not available for hyperbolic relaxation systems in domains with boundary in the literature.

A special well investigated problem is the semilinear relaxation approximation to the boundary value problem for a scalar quasilinear equation, see [11, 14, 10, 12], and $[4,1]$ for related numerical considerations.

For the strong solutions of the (KD) boundary value problem with the entropic variables, we obtain a symmetric hyperbolic system endowed with a flat equilibrium manifold. This basic structure of numerous relaxation systems is explained and used for the global existence of smooth solutions in [6] and for asymptotic behavior in [2]. For connected works see also [18, 9].

The study of the three-dimensional case is the subject of a work in progress [3]. In this case the previous properties are still valid: there is no boundary layer and with the entropic variables, the equilibrium manifold is flat. On the other hand, the boundary is characteristic for both problems, with a two-dimensional kernel for the Kerr problem, and a three-dimensional kernel for the Kerr-Debye problem. We must then take into account the nonlinear conservation equations $\operatorname{div} D=\operatorname{div} B=0$, which is irrelevant for the one-dimensional case.

Section 3 is devoted to the proof of Theorem 2.3. Using energy estimates, we bound the time derivatives of $W_{\varepsilon}$. The boundary is characteristic so we cannot directly obtain the bounds for the space derivatives. Since $\operatorname{Ker} A_{1}=\mathbb{R}(0,0,1)$ with the first two equations, we can express and estimate the space derivatives for $U_{\varepsilon}$. Estimates for $v_{\varepsilon}$ are obtained solving the third equation by the Duhamel formula.

We prove Theorem 2.4 in Section 4.

\section{Proof of Theorem 2.3}

Proof. We denote by · the canonical scalar product on $\mathbb{R}^{3}$ and by $|\cdot|$ the associated Euclidean norm.

For the convenience of the reader, we rewrite System (2.12) omitting the dependance on $\varepsilon$. Let $W$ be a solution to the problem

$$
\left\{\begin{array}{l}
A_{0}(W) \partial_{t} W+A_{1} \partial_{x} W=-\frac{2}{\varepsilon} Q(W)+G \text { on }\left[0, T_{\varepsilon}^{*}\right] \times \mathbb{R}^{+}, \\
W(0, x)=0 \text { on } \mathbb{R}^{+} \\
(h+a e)(t, 0)=0 \text { on }\left[0, T_{\varepsilon}^{*}\right] .
\end{array}\right.
$$

Here we denote by $T_{\varepsilon}^{*} \in[0,+\infty]$ the lifespan of $W$ given by [5]. We prove the result when

$$
\|W\|_{L^{\infty}\left(0, T_{\varepsilon}^{*} \times \mathbb{R}^{+}\right)}=+\infty .
$$


If this assumption is not satisfied, we use the extension Theorem in [5] with analogous arguments as below.

Let $M$ be a positive constant large enough with respect to the $L^{\infty}$-norm of the initial data. Consider the regular solution $W=(U, v)$ to problem (3.1), as given by Proposition 2.2. Define $T_{\varepsilon}>0$ as

$$
T_{\varepsilon}=\max \left\{T \leq T_{\varepsilon}^{*},\|W\|_{L^{\infty}\left([0, T] \times \mathbb{R}^{+}\right)}<M\right\},
$$

that is $T_{\varepsilon}$ is the first time such that the $L^{\infty}$-norm of $W$ reaches the given bound $M$. We recall that the solution $W=(U, v)$ satisfies:

$$
\partial_{t}^{i} W \in \mathcal{C}^{0}\left(\left[0, T_{\varepsilon}\right] ; H^{2-i}\left(\mathbb{R}^{+}\right)\right) \text {for } i=0,1,2 .
$$

Furthermore we recall that

$$
e^{2}+2 v \geq 0 \text { on }\left[0, T_{\varepsilon}\right] \times \mathbb{R}^{+} .
$$

From (3.2) and (3.4), by definition of $A_{0}$ we have

$$
\forall \xi \in \mathbb{R}^{3}, A_{0}(W) \xi \cdot \xi \geq \frac{1}{M^{2}+2}|\xi|^{2} .
$$

We measure the boundary value lifting $G$ by the quantities $\gamma$ and $\Gamma$ defined by:

$$
\gamma(t)=\left(\|G(t)\|_{L^{2}\left(\mathbb{R}^{+}\right)}^{2}+\left\|\partial_{t} G(t)\right\|_{L^{2}\left(\mathbb{R}^{+}\right)}^{2}+\left\|\partial_{t t} G(t)\right\|_{L^{2}\left(\mathbb{R}^{+}\right)}^{2}+\left\|\partial_{x} G(t)\right\|_{L^{2}\left(\mathbb{R}^{+}\right)}^{2}\right)^{\frac{1}{2}},
$$

and

$$
\Gamma(t)=\sup _{s \in[0, t]} \gamma(s) .
$$

For $t \in\left[0, T_{\varepsilon}\right]$ we define $\varphi$ and $\Phi$ by

$$
\begin{gathered}
\varphi(t)=\left(\|W(t)\|_{L^{2}\left(\mathbb{R}^{+}\right)}^{2}+\left\|\partial_{t} W(t)\right\|_{L^{2}\left(\mathbb{R}^{+}\right)}^{2}+\left\|\partial_{t t} W(t)\right\|_{L^{2}\left(\mathbb{R}^{+}\right)}^{2}\right)^{\frac{1}{2}}, \\
\Phi(t)=\sup _{s \in[0, t]} \varphi(s) .
\end{gathered}
$$

We first prove the following result.

Lemma 3.1. There exists a constant $K_{1}$, independent of $M$, such that

$$
\forall \varepsilon>0, \forall t \in\left[0, T_{\varepsilon}\right], \Phi(t)^{2} \leq K_{1}\left(2+M^{2}\right) \int_{0}^{t}\left(1+(\Phi(s))^{5}+(\Gamma(s))^{5}\right) d s .
$$

Proof. First step: $L^{2}$ estimate

We take the inner product of Equation (3.1) with $W$ and we obtain

$$
\begin{aligned}
& \frac{1}{2} \frac{d}{d t} \int_{\mathbb{R}^{+}} A_{0}(W) W \cdot W d x+\frac{2}{\varepsilon} \int_{\mathbb{R}^{+}}|v|^{2} d x+a|e(t, 0)|^{2} \\
= & \int_{\mathbb{R}^{+}} G \cdot W+\frac{1}{2} \int_{\mathbb{R}^{+}} \partial_{t}\left(A_{0}(W)\right) W \cdot W d x,
\end{aligned}
$$


where

$$
\partial_{t}\left(A_{0}(W)\right)=\left(\begin{array}{ccc}
6 e \partial_{t} e+2 \partial_{t} v & 0 & 2 \partial_{t} e \\
0 & 0 & 0 \\
2 \partial_{t} e & 0 & 0
\end{array}\right)
$$

We have

$$
\begin{aligned}
& \left|\int_{\mathbb{R}^{+}} \partial_{t}\left(A_{0}(W)\right) W \cdot W d x\right| \\
\leq & \left\|\partial_{t}\left(A_{0}(W)\right)\right\|_{L^{\infty}\left(\mathbb{R}^{+}\right)}\|W\|_{L^{2}\left(\mathbb{R}^{+}\right)}^{2} \\
\leq & C\left(1+\|e\|_{L^{\infty}\left(\mathbb{R}^{+}\right)}\right)\left(\left\|\partial_{t} e\right\|_{L^{\infty}\left(\mathbb{R}^{+}\right)}+\left\|\partial_{t} v\right\|_{L^{\infty}\left(\mathbb{R}^{+}\right)}\right)\|W\|_{L^{2}\left(\mathbb{R}^{+}\right)}^{2},
\end{aligned}
$$

thus we obtain

$$
\begin{aligned}
\frac{1}{2} \frac{d}{d t} \int_{\mathbb{R}^{+}} A_{0}(W) W \cdot W d x & +\frac{2}{\varepsilon} \int_{\mathbb{R}^{+}}|v|^{2} d x+a|e(t, 0)|^{2} \leq\|G\|_{L^{2}\left(\mathbb{R}^{+}\right)}\|W\|_{L^{2}\left(\mathbb{R}^{+}\right)} \\
& +C\left(1+\|e\|_{L^{\infty}\left(\mathbb{R}^{+}\right)}\right)\left(\left\|\partial_{t} e\right\|_{L^{\infty}\left(\mathbb{R}^{+}\right)}+\left\|\partial_{t} v\right\|_{L^{\infty}\left(\mathbb{R}^{+}\right)}\right)\|W\|_{L^{2}\left(\mathbb{R}^{+}\right)}^{2} .
\end{aligned}
$$

\section{Second step: estimate on $\partial_{t} W$}

We can derivate the system (3.1) with respect to $t$. We obtain

$$
\left\{\begin{array}{l}
A_{0}(W) \partial_{t t} W+\partial_{t}\left(A_{0}(W)\right) \partial_{t} W+A_{1} \partial_{t x} W=-\frac{2}{\varepsilon} Q\left(\partial_{t} W\right)+\partial_{t} G \text { on }\left[0, T_{\varepsilon}\right] \times \mathbb{R}^{+}, \\
\partial_{t} W(0, x)=0 \text { on } \mathbb{R}^{+}, \\
\left(\partial_{t} h+a \partial_{t} e\right)(t, 0)=0 \text { on }\left[0, T_{\varepsilon}\right] .
\end{array}\right.
$$

Taking the inner product of (3.13) with $\partial_{t} W$, we obtain

$$
\begin{array}{r}
\frac{1}{2} \frac{d}{d t} \int_{\mathbb{R}^{+}} A_{0}(W) \partial_{t} W \cdot \partial_{t} W d x+\frac{2}{\varepsilon} \int_{\mathbb{R}^{+}}\left|\partial_{t} v\right|^{2} d x+a\left|\partial_{t} e(t, 0)\right|^{2}=\int_{\mathbb{R}^{+}} \partial_{t} G \cdot \partial_{t} W \\
-\frac{1}{2} \int_{\mathbb{R}^{+}} \partial_{t}\left(A_{0}(W)\right) \partial_{t} W \cdot \partial_{t} W d x,
\end{array}
$$

and thus there exists a constant $C$ such that

$$
\begin{aligned}
& \frac{1}{2} \frac{d}{d t} \int_{\mathbb{R}^{+}} A_{0}(W) \partial_{t} W \cdot \partial_{t} W d x+\frac{2}{\varepsilon} \int_{\mathbb{R}^{+}}\left|\partial_{t} v\right|^{2} d x+a\left|\partial_{t} e(t, 0)\right|^{2} \\
\leq & \left\|\partial_{t} G\right\|_{L^{2}\left(\mathbb{R}^{+}\right)}\left\|\partial_{t} W\right\|_{L^{2}\left(\mathbb{R}^{+}\right)} \\
& +C\left(1+\|e\|_{L^{\infty}\left(\mathbb{R}^{+}\right)}\right)\left(\left\|\partial_{t} e\right\|_{L^{\infty}\left(\mathbb{R}^{+}\right)}+\left\|\partial_{t} v\right\|_{L^{\infty}\left(\mathbb{R}^{+}\right)}\right)\left\|\partial_{t} W\right\|_{L^{2}\left(\mathbb{R}^{+}\right)}^{2} .
\end{aligned}
$$

Third step: estimate on $\partial_{t t} W$

We can derivate System (3.13) with respect to $t$. We obtain

$$
\begin{array}{r}
\frac{1}{2} \frac{d}{d t} \int_{\mathbb{R}^{+}} A_{0}(W) \partial_{t t} W \cdot \partial_{t t} W d x+\frac{2}{\varepsilon} \int_{\mathbb{R}^{+}}\left|\partial_{t t} v\right|^{2} d x+a\left|\partial_{t t} e(t, 0)\right|^{2}=\int_{\mathbb{R}^{+}} \partial_{t t} G \cdot \partial_{t t} W \\
-\frac{3}{2} \int_{\mathbb{R}^{+}} \partial_{t}\left(A_{0}(W)\right) \partial_{t t} W \cdot \partial_{t t} W d x-\int_{\mathbb{R}^{+}} \partial_{t t}\left(A_{0}(W)\right) \partial_{t} W \cdot \partial_{t t} W .
\end{array}
$$


Now we have:

$$
\partial_{t t}\left(A_{0}(W)\right)=\left(\begin{array}{ccc}
6\left(\partial_{t} e\right)^{2}+6 e \partial_{t t} e+2 \partial_{t t} v & 0 & 2 \partial_{t t} e \\
0 & 0 & 0 \\
2 \partial_{t t} e & 0 & 0
\end{array}\right)
$$

thus

$$
\begin{aligned}
& \left\|\partial_{t t}\left(A_{0}(W)\right) \partial_{t} W\right\|_{L^{2}\left(\mathbb{R}^{+}\right)} \\
\leq & C\left(1+\|e\|_{L^{\infty}\left(\mathbb{R}^{+}\right)}\right)\left(\left\|\partial_{t} e\right\|_{L^{\infty}\left(\mathbb{R}^{+}\right)}+\left\|\partial_{t} v\right\|_{L^{\infty}\left(\mathbb{R}^{+}\right)}\right)\left\|\partial_{t t} W\right\|_{L^{2}\left(\mathbb{R}^{+}\right)} \\
& +C\left\|\partial_{t} e\right\|_{L^{\infty}\left(\mathbb{R}^{+}\right)}^{2}\left\|\partial_{t} e\right\|_{L^{2}\left(\mathbb{R}^{+}\right)} .
\end{aligned}
$$

Therefore

$$
\begin{aligned}
& \frac{1}{2} \frac{d}{d t} \int_{\mathbb{R}^{+}} A_{0}(W) \partial_{t t} W \cdot \partial_{t t} W d x+\frac{2}{\varepsilon} \int_{\mathbb{R}^{+}}\left|\partial_{t t} v\right|^{2} d x+a\left|\partial_{t t} e(t, 0)\right|^{2} \\
\leq & \left\|\partial_{t t} G\right\|_{L^{2}\left(\mathbb{R}^{+}\right)}\left\|\partial_{t t} W\right\|_{L^{2}\left(\mathbb{R}^{+}\right)} \\
& +C\left(1+\|e\|_{L^{\infty}\left(\mathbb{R}^{+}\right)}\right)\left(\left\|\partial_{t} e\right\|_{L^{\infty}\left(\mathbb{R}^{+}\right)}+\left\|\partial_{t} v\right\|_{L^{\infty}\left(\mathbb{R}^{+}\right)}\right)\left\|\partial_{t t} W\right\|_{L^{2}\left(\mathbb{R}^{+}\right)}^{2} \\
& +C\left\|\partial_{t} e\right\|_{L^{\infty}\left(\mathbb{R}^{+}\right)}^{2}\left\|\partial_{t} e\right\|_{L^{2}\left(\mathbb{R}^{+}\right)}\left\|\partial_{t t} W\right\|_{L^{2}\left(\mathbb{R}^{+}\right)} .
\end{aligned}
$$

Fourth step: $L^{\infty}$ estimates for $\partial_{t} e$ and $\partial_{t} v$

We recall the equations satisfied by $W=(e, h, v)$ :

$$
\begin{gathered}
\left(3 e^{2}+2 v+1\right) \partial_{t} e+2 e \partial_{t} v+\partial_{x} h=g_{1}, \\
\partial_{t} h+\partial_{x} e=g_{2} \\
e \partial_{t} e+\partial_{t} v=-\frac{1}{\varepsilon} v
\end{gathered}
$$

From (3.17) we have

$$
\left\|\partial_{x} e\right\|_{L^{2}\left(\mathbb{R}^{+}\right)} \leq \varphi+\|G\|_{L^{2}\left(\mathbb{R}^{+}\right)} \leq \varphi+\gamma,
$$

where $\varphi$ is defined by (3.8) and $\gamma$ is defined by (3.6). Thus

$$
\|e\|_{H^{1}\left(\mathbb{R}^{+}\right)} \leq 2 \varphi+\gamma,
$$

and by Sobolev injection

$$
\|e\|_{L^{\infty}\left(\mathbb{R}^{+}\right)} \leq C(\varphi+\gamma) .
$$

Derivating (3.17) with respect to $t$ we obtain that

$$
\left\|\partial_{x} \partial_{t} e\right\|_{L^{2}\left(\mathbb{R}^{+}\right)} \leq \varphi+\left\|\partial_{t} G\right\|_{L^{2}\left(\mathbb{R}^{+}\right)},
$$

so

$$
\left\|\partial_{t} e\right\|_{H^{1}\left(\mathbb{R}^{+}\right)} \leq 2 \varphi+\left\|\partial_{t} G\right\|_{L^{2}\left(\mathbb{R}^{+}\right)}
$$


and by Sobolev injections there exists a constant $C$ such that

$$
\left\|\partial_{t} e\right\|_{L^{\infty}\left(\mathbb{R}^{+}\right)} \leq C(\varphi+\gamma) .
$$

Now we solve Equation (3.18) with the Duhamel formula:

$$
v(t, x)=-\int_{0}^{t} \exp \left(\frac{s-t}{\varepsilon}\right) e(s, x) \partial_{t} e(s, x) d s,
$$

so

$$
\begin{aligned}
\|v(t, .)\|_{L^{\infty}\left(\mathbb{R}^{+}\right)} & \leq \int_{0}^{t} \exp \left(\frac{s-t}{\varepsilon}\right)\|e(s, .)\|_{L^{\infty}\left(\mathbb{R}^{+}\right)}\left\|\partial_{t} e(s, .)\right\|_{L^{\infty}\left(\mathbb{R}^{+}\right)} d s \\
& \leq C \int_{0}^{t} \exp \left(\frac{s-t}{\varepsilon}\right)(\varphi(s)+\gamma(s))^{2} d s \\
& \leq C(\Phi(t)+\Gamma(t))^{2} \varepsilon,
\end{aligned}
$$

where $\Phi$ is defined in (3.9) and $\Gamma$ is defined by (3.7). Using Equation (3.18) we obtain then that

$$
\begin{aligned}
\left\|\partial_{t} v\right\|_{L^{\infty}\left(\mathbb{R}^{+}\right)} & \leq \frac{1}{\varepsilon}\|v\|_{L^{\infty}\left(\mathbb{R}^{+}\right)}+\left\|e \partial_{t} e\right\|_{L^{\infty}\left(\mathbb{R}^{+}\right)} \\
& \leq C(\Phi(t)+\Gamma(t))^{2} .
\end{aligned}
$$

Fifth step: end of the proof of Lemma 3.1

We sum up inequalities (3.12), (3.14) and (3.15). Using (3.23) and (3.24) we obtain that there exists a constant $K_{1}$ independent of $\varepsilon, M$ and $t \in\left[0, T_{\varepsilon}\right]$ such that

$$
\begin{aligned}
& \frac{1}{2} \frac{d}{d t} \int_{\mathbb{R}^{+}}\left(A_{0}(W) W \cdot W d x+A_{0}(W) \partial_{t} W \cdot \partial_{t} W d x+A_{0}(W) \partial_{t t} W \cdot \partial_{t t} W\right) d x \\
& +\frac{2}{\varepsilon} \int_{\mathbb{R}^{+}}\left(|v|^{2} d x+\left|\partial_{t} v\right|^{2} d x+\left|\partial_{t t} v\right|^{2}\right) d x+a\left(|e(t, 0)|^{2}+\left|\partial_{t} e(t, 0)\right|^{2}+\left|\partial_{t t} e(t, 0)\right|^{2}\right) \\
& \quad \leq C \gamma \varphi+C(1+\varphi+\gamma)\left(\varphi+\gamma+(\Phi+\Gamma)^{2}\right) \varphi^{2}+C(\varphi+\gamma)^{2} \varphi^{2} \\
& \quad \leq K_{1}\left(1+\Phi^{5}+\Gamma^{5}\right) .
\end{aligned}
$$

We integrate this inequality on $[0, t]$ for $t \in\left[0, T_{\varepsilon}\right]$ and we have by (3.5) that

$$
\forall t \in\left[0, T_{\varepsilon}\right], \varphi^{2}(t) \leq K_{1}\left(2+M^{2}\right) \int_{0}^{t}\left(1+\Phi(s)^{5}+\Gamma(s)^{5}\right) d s .
$$

So we obtain Lemma 3.1.

Lemma 3.1 provides estimates on the time derivatives of $W$. The space derivatives can not be obtained by the same method since it is impossible to derivate the system (KD) with respect to the normal variable $x$. We deduce estimates on the space derivatives from the equations as we will see in the following lemma. 
Lemma 3.2. There exists $K_{2}$ such that for all $t<T_{\varepsilon}^{*}$,

$$
\begin{aligned}
& \|U\|_{H^{2}\left(\mathbb{R}^{+}\right)} \leq K_{2}\left(\Gamma+\Phi+\Gamma^{3}+\Phi^{3}\right), \\
& \|v\|_{H^{1}\left(\mathbb{R}^{+}\right)} \leq K_{2}\left(\Gamma^{2}+\Phi^{2}\right) \varepsilon \\
& \left\|\partial_{t} v\right\|_{H^{1}\left(\mathbb{R}^{+}\right)} \leq K_{2}\left(\Gamma^{2}+\Phi^{2}\right) \\
& \|W\|_{L^{\infty}\left(\mathbb{R}^{+}\right)} \leq K_{2}\left(\Gamma+\Phi+\Gamma^{3}+\Phi^{3}\right) .
\end{aligned}
$$

Proof. We recall that

$$
v(t, x)=-\int_{0}^{t} \exp \left(\frac{s-t}{\varepsilon}\right) e(s, x) \partial_{t} e(s, x) d s
$$

thus

$$
\|v(t, .)\|_{H^{1}\left(\mathbb{R}^{+}\right)} \leq C \int_{0}^{t} \exp \left(\frac{s-t}{\varepsilon}\right)\|e(s, .)\|_{H^{1}\left(\mathbb{R}^{+}\right)}\left\|\partial_{t} e(s, .)\right\|_{H^{1}\left(\mathbb{R}^{+}\right)} d s,
$$

thus by Estimates (3.22) and (3.20), we have

$$
\|v(t, .)\|_{H^{1}\left(\mathbb{R}^{+}\right)} \leq C(\Phi(t)+\Gamma(t))^{2} \varepsilon .
$$

From (3.18) we obtain

$$
\left\|\partial_{t} v(t, .)\right\|_{H^{1}\left(\mathbb{R}^{+}\right)} \leq \frac{1}{\varepsilon}\|v(t, .)\|_{H^{1}\left(\mathbb{R}^{+}\right)}+\|e\|_{H^{1}\left(\mathbb{R}^{+}\right)}\left\|\partial_{t} e\right\|_{H^{1}\left(\mathbb{R}^{+}\right)},
$$

thus

$$
\left\|\partial_{t} v(t, .)\right\|_{H^{1}\left(\mathbb{R}^{+}\right)} \leq C(\Phi(t)+\Gamma(t))^{2} .
$$

Now from Equation (3.16) we estimate $\partial_{x} h$ in the following way:

$$
\begin{array}{r}
\left\|\partial_{x} h\right\|_{H^{1}\left(\mathbb{R}^{+}\right) \leq \|} g_{1}\left\|_{H^{1}\left(\mathbb{R}^{+}\right)}+\right\| 3 e^{2}+2 v\left\|_{H^{1}\left(\mathbb{R}^{+}\right)}\right\| \partial_{t} e \|_{H^{1}\left(\mathbb{R}^{+}\right)} \\
+\left\|\partial_{t} e\right\|_{H^{1}\left(\mathbb{R}^{+}\right)}+2\|e\|_{H^{1}\left(\mathbb{R}^{+}\right)}\left\|\partial_{t} v\right\|_{H^{1}\left(\mathbb{R}^{+}\right)}
\end{array}
$$

and thus there exists a constant $C$ such that

$$
\|h\|_{H^{2}\left(\mathbb{R}^{+}\right)} \leq C\left(\Gamma+\Phi+\Gamma^{3}+\Phi^{3}\right) .
$$

We derivate (3.17) with respect to $x$ :

$$
\partial_{x x} e=\partial_{x} g_{2}-\partial_{t} \partial_{x} h
$$

Thus

$$
\left\|\partial_{x x} e\right\|_{L^{2}\left(\mathbb{R}^{+}\right)}=\gamma+\left\|\partial_{t} \partial_{x} h\right\|_{L^{2}\left(\mathbb{R}^{+}\right)} .
$$

Derivating (3.16) with respect to $t$ we obtain: 


$$
\begin{aligned}
& \left\|\partial_{t} \partial_{x} h\right\|_{L^{2}\left(\mathbb{R}^{+}\right)} \\
\leq & \left\|3 e^{2}+2 v+1\right\|_{L^{\infty}\left(\mathbb{R}^{+}\right)}\left\|\partial_{t t} e\right\|_{L^{2}\left(\mathbb{R}^{+}\right)}+\left\|6 e \partial_{t} e+2 \partial_{t} v\right\|_{L^{2}\left(\mathbb{R}^{+}\right)}\left\|\partial_{t} e\right\|_{L^{\infty}\left(\mathbb{R}^{+}\right)} \\
& +2\left\|\partial_{t} e\right\|_{L^{\infty}\left(\mathbb{R}^{+}\right)}\left\|\partial_{t} v\right\|_{L^{2}\left(\mathbb{R}^{+}\right)}+2\left\|\partial_{t} e\right\|_{L^{\infty}\left(\mathbb{R}^{+}\right)}\left\|\partial_{t t} v\right\|_{L^{2}\left(\mathbb{R}^{+}\right)} \\
& +\left\|\partial_{t} g_{1}\right\|_{L^{2}\left(\mathbb{R}^{+}\right)} \\
\leq & C\left(\Gamma+\Phi+\Phi^{3}+\Gamma^{3}\right),
\end{aligned}
$$

using (3.21), (3.23), (3.24) and (3.25).

So we have obtained

$$
\|e\|_{H^{2}\left(\mathbb{R}^{+}\right)} \leq C\left(\Gamma+\Phi+\Phi^{3}+\Gamma^{3}\right) .
$$

This concludes the proof of Lemma 3.2.

\section{Proof of Theorem 2.3}

We fix $M=3 K_{2}\left(\Gamma_{\infty}+\Gamma_{\infty}^{3}\right)$, where $K_{2}$ is given by Lemma 3.2, and where $\Gamma_{\infty}=\sup _{\mathbb{R}^{+}} \Gamma$.

Let us introduce $\xi$ the solution of

$$
\left\{\begin{array}{l}
\xi^{\prime}=K_{1}\left(2+M^{2}\right)\left(1+\xi^{\frac{5}{2}}+\Gamma_{\infty}^{5}\right), \\
\xi(0)=0,
\end{array}\right.
$$

defined on the maximal interval $\left[0, T_{1}\right]$, where $K_{1}$ is given by Lemma 3.1. By comparison results, from Estimate (3.10) we have

$$
\forall t \in\left[0, \min \left(T_{1}, T_{\varepsilon}\right)\right], \Phi^{2}(t) \leq \xi(t) .
$$

From Lemma 3.2 we have then that

$$
\|W\|_{L^{\infty}\left(\mathbb{R}^{+}\right)} \leq K_{2}\left(\Gamma+\xi^{\frac{1}{2}}+\Gamma^{3}+\xi^{\frac{3}{2}}\right) .
$$

The map $\xi$ is continuous and $\xi(0)=0$, thus there exists a time $\tilde{T}$ with $0<\tilde{T} \leq T_{1}$ such that

$$
K_{2}\left(\xi^{\frac{1}{2}}(\tilde{T})+\xi^{\frac{3}{2}}(\tilde{T})\right) \leq \frac{M}{3}
$$

For all $t \leq \tilde{T}$, we then have:

$$
K_{2}\left(\Gamma+\xi^{\frac{1}{2}}+\Gamma^{3}+\xi^{\frac{3}{2}}\right) \leq \frac{2 M}{3} .
$$

Thus $\tilde{T}<T_{\varepsilon}$ because if $T_{\varepsilon} \leq \tilde{T}$ then $\|W(t)\|_{L^{\infty}\left(\mathbb{R}^{+}\right)} \leq \frac{2 M}{3}$ for all $t \leq T_{\varepsilon}$, which is contradictory to the fact that $\left\|W\left(T_{\varepsilon}\right)\right\|_{L^{\infty}\left(\mathbb{R}^{+}\right)}=M$ (see (3.2)).

It remains to obtain uniform $H^{2}$ estimate on $v$. We have

$$
v(t, x)=-\frac{1}{2} \int_{0}^{t} \exp \left(\frac{s-t}{\varepsilon}\right) \partial_{t}\left(e(s, x)^{2}\right) d s,
$$


thus with an integration by parts we obtain

$$
v(t, x)=-\frac{1}{2}\left[\exp \left(\frac{s-t}{\varepsilon}\right) e(s, x)^{2}\right]_{0}^{t}+\frac{1}{2 \varepsilon} \int_{0}^{t} \exp \left(\frac{s-t}{\varepsilon}\right) e(s, x)^{2} d s .
$$

So we obtain that there exists a constant $C$ independent of $\varepsilon$ such that for $t \leq \tilde{T}$

$$
\|v(t, .)\|_{H^{2}\left(\mathbb{R}^{+}\right)} \leq C\|e\|_{L^{\infty}\left([0, t] ; H^{2}\left(\mathbb{R}^{+}\right)\right)}^{2} .
$$

This concludes the proof of Theorem 2.3.

\section{Proof of Theorem 2.4}

Proof. Let us consider $U=(e, h)$ the solution of the $(\mathrm{K})$ boundary value problem (2.11) defined on the time interval $\left[0, T^{*}\right]$ given by Proposition 2.1. We recall that we denote by $W_{\varepsilon}=\left(U_{\varepsilon}, v_{\varepsilon}\right)$ the solution of the (KD) boundary value problem $(2.12)$ given by Proposition 2.2 , with $U_{\varepsilon}=\left(e_{\varepsilon}, h_{\varepsilon}\right)$.

Set $R_{\varepsilon}=\frac{1}{\varepsilon}\left(U_{\varepsilon}-U\right)=\left(r_{\varepsilon}, s_{\varepsilon}\right)$. The remainder term $R_{\varepsilon}$ satisfies

$$
\left\{\begin{array}{l}
\left(3 e_{\varepsilon}^{2}+2 v_{\varepsilon}+1\right) \partial_{t} r_{\varepsilon}+\partial_{x} s_{\varepsilon}+3\left(e_{\varepsilon}+e\right)\left(\partial_{t} e\right) r_{\varepsilon}=-\frac{2}{\varepsilon} e_{\varepsilon} \partial_{t} v_{\varepsilon}-\frac{2}{\varepsilon} v_{\varepsilon} \partial_{t} e, \\
\partial_{t} s_{\varepsilon}+\partial_{x} r_{\varepsilon}=0
\end{array}\right.
$$

with the initial and boundary conditions:

$$
\left\{\begin{array}{l}
s_{\varepsilon}=e_{\varepsilon}=0 \text { for } t=0 \\
s_{\varepsilon}+a r_{\varepsilon}=0 \text { for } x=0 .
\end{array}\right.
$$

In order to estimate the right hand side term we recall that from (2.12) we have

$$
\frac{1}{\varepsilon} \partial_{t} v_{\varepsilon}=-\partial_{t t} v_{\varepsilon}-\left(\partial_{t} e_{\varepsilon}\right)^{2}-e_{\varepsilon} \partial_{t t} e_{\varepsilon}
$$

Using Theorem 2.3, for all $T \leq \tilde{T}$, there exists a constant $K$ independent of $\varepsilon$ such that

$$
\left\|\partial_{t} v_{\varepsilon}\right\|_{L^{2}\left(\mathbb{R}^{+}\right)} \leq K \varepsilon .
$$

We take the inner product of (4.1) with $R_{\varepsilon}$ to obtain

$$
\begin{gathered}
\frac{1}{2} \frac{d}{d t} \int_{\mathbb{R}^{+}}\left(\left(3 e_{\varepsilon}^{2}+2 v_{\varepsilon}+1\right) r_{\varepsilon}^{2}+s_{\varepsilon}^{2}\right)+a\left(s_{\varepsilon}(t, 0)\right)^{2}=\frac{1}{2} \int_{\mathbb{R}^{+}}\left(6 e_{\varepsilon} \partial_{t} e_{\varepsilon}+2 \partial_{t} v_{\varepsilon}\right) r_{\varepsilon}^{2} \\
-\int_{\mathbb{R}^{+}} 3\left(e_{\varepsilon}+e\right)\left(\partial_{t} e\right) r_{\varepsilon}^{2}-\frac{2}{\varepsilon} \int_{\mathbb{R}^{+}} e_{\varepsilon}\left(\partial_{t} v_{\varepsilon}\right) r_{\varepsilon}-\frac{2}{\varepsilon} \int_{\mathbb{R}^{+}} v_{\varepsilon}\left(\partial_{t} e\right) r_{\varepsilon} .
\end{gathered}
$$

So, there exists a constant $C$ such that

$$
\begin{aligned}
& \frac{1}{2} \frac{d}{d t} \int_{\mathbb{R}^{+}}\left(\left(3 e_{\varepsilon}^{2}+2 v_{\varepsilon}+1\right) r_{\varepsilon}^{2}+s_{\varepsilon}^{2}\right) \\
\leq & C\left(\left\|e_{\varepsilon}\right\|_{L^{\infty}\left(\mathbb{R}^{+}\right)}\left\|\partial_{t} e_{\varepsilon}\right\|_{L^{\infty}\left(\mathbb{R}^{+}\right)}+\left\|\partial_{t} v_{\varepsilon}\right\|_{L^{\infty}\left(\mathbb{R}^{+}\right)}\right)\left\|r_{\varepsilon}\right\|_{L^{2}\left(\mathbb{R}^{+}\right)}^{2} \\
& +C\left(\left\|e_{\varepsilon}\right\|_{L^{\infty}\left(\mathbb{R}^{+}\right)}\left\|\partial_{t} e\right\|_{L^{\infty}\left(\mathbb{R}^{+}\right)}+\|e\|_{L^{\infty}\left(\mathbb{R}^{+}\right)}\left\|\partial_{t} e\right\|_{L^{\infty}\left(\mathbb{R}^{+}\right)}\right)\left\|r_{\varepsilon}\right\|_{L^{2}\left(\mathbb{R}^{+}\right)}^{2} \\
& +\frac{2}{\varepsilon}\left\|e_{\varepsilon}\right\|_{L^{\infty}\left(\mathbb{R}^{+}\right)}\left\|\partial_{t} v_{\varepsilon}\right\|_{L^{2}\left(\mathbb{R}^{+}\right)}\left\|r_{\varepsilon}\right\|_{L^{2}\left(\mathbb{R}^{+}\right)}+\frac{2}{\varepsilon}\left\|\partial_{t} e\right\|_{L^{\infty}\left(\mathbb{R}^{+}\right)}\left\|v_{\varepsilon}\right\|_{L^{2}\left(\mathbb{R}^{+}\right)}\left\|r_{\varepsilon}\right\|_{L^{2}\left(\mathbb{R}^{+}\right)} .
\end{aligned}
$$


From the estimates of Theorem 2.3, from the properties of $e$ (see Proposition 2.1) and from (4.3), there exists a constant $K$ such that for all $T \leq \min \left(\tilde{T}, T^{*}\right)$,

$$
\frac{1}{2} \frac{d}{d t} \int_{\mathbb{R}^{+}}\left(\left(3 e_{\varepsilon}^{2}+2 v_{\varepsilon}+1\right) r_{\varepsilon}^{2}+s_{\varepsilon}^{2}\right) \leq K\left(1+\left\|r_{\varepsilon}\right\|_{L^{2}\left(\mathbb{R}^{+}\right)}^{2}\right) .
$$

We integrate this equation from 0 to $t$ and we obtain, using (3.5),

$$
\left\|r_{\varepsilon}(t)\right\|_{L^{2}\left(\mathbb{R}^{+}\right)}^{2}+\left\|s_{\varepsilon}(t)\right\|_{L^{2}\left(\mathbb{R}^{+}\right)}^{2} \leq K\left(M^{2}+2\right) \int_{0}^{t}\left(1+\left\|r_{\varepsilon}(\tau)\right\|_{L^{2}\left(\mathbb{R}^{+}\right)}^{2}\right) d \tau .
$$

We conclude the proof of Theorem 2.4 by the Gronwall Lemma.

\section{REFERENCES}

[1] Denise Aregba-Driollet and Vuk Milišić, Kinetic approximation of a boundary value problem for conservation laws, Numer. Math., 97(4), 595-633, 2004.

[2] Stefano Bianchini, Bernard Hanouzet and Roberto Natalini, Asymptotic Behaviour of Smooth Solutions for Partially Dissipative Hyperbolic Systems with a Convex Entropy, Comm. Pure Appl. Math., preprint, 2005.

[3] Gilles Carbou and Bernard Hanouzet, Relaxation Approximation of the Kerr Model for the three dimensional initial-boundary value problem, in preparation.

[4] A. Chalabi and D. Seghir, Convergence of relaxation schemes for initial boundary value problems for conservation laws, Comput. Math. Appl., 43(8-9), 1079-1093, 2002.

[5] Olivier Guès, Problème mixte hyperbolique quasi-linéaire caractéristique, Comm. Partial Differential Equations, 15(5), 595-645, 1990.

[6] B. Hanouzet and R. Natalini, Global existence of smooth solutions for partially dissipative hyperbolic systems with a convex entropy, Arch. Ration. Mech. Anal., 169(2), 89-117, 2003.

[7] Bernard Hanouzet and Philippe Huynh, Approximation par relaxation d'un système de Maxwell non linéaire, C. R. Acad. Sci. Paris Sér. I Math., 330(3), 193-198, 2000.

[8] Philippe Huynh, Etudes théorique et numérique de modèles de Kerr, Thèse, Université Bordeaux 1, 1999.

[9] S. Kawashima and W.-A. Yong, Dissipative structure and entropy for hyperbolic systems of balance laws, Arch. Ration. Mech. Anal., 174, 345-364, 2004.

[10] Wendy Kress, Asymptotic behavior of hyperbolic boundary value problems with relaxation term, In Hyperbolic problems: theory, numerics, applications, I, II, Magdeburg, 2000, Internat. Ser. Numer. Math., 141, 633-642, 2001.

[11] Hailiang Liu and Wen-An Yong, Time-asymptotic stability of boundary-layers for a hyperbolic relaxation system, Comm. Partial Differential Equations, 26(7-8), 1323-1343, 2001.

[12] Vuk Milišić, Stability and convergence of discrete kinetic approximations to an initial-boundary value problem for conservation laws, Proc. Amer. Math. Soc., 131(6), 1727-1737, (electronic), 2003.

[13] Roberto Natalini, Recent results on hyperbolic relaxation problems, Analysis of systems of conservation laws, Aachen, 1997, Chapman \& Hall/CRC Monogr. Surv. Pure Appl. Math., Chapman \& Hall/CRC, Boca Raton, FL, 99, 128-198, 1999.

[14] Roberto Natalini and Andrea Terracina, Convergence of a relaxation approximation to a boundary value problem for conservation laws, Comm. Partial Differential Equations, 26(7-8), 1235-1252, 2001.

[15] Y.- R. Shen, The Principles of Nonlinear Optics, Wiley Interscience, 1994.

[16] Wen-An Yong, Boundary conditions for hyperbolic systems with stiff source terms, Indiana Univ. Math. J., 48(1), 115-137, 1999.

[17] Wen-An Yong, Singular perturbations of first-order hyperbolic systems with stiff source terms, J. Differential Equations, 155(1), 89-132, 1999.

[18] Wen-An Yong, Entropy and Global Existence for Hyperbolic Balance Laws, Arch. Ration. Mech. Anal., 172(2), 247-266, 2004.

[19] R.-W. Ziolkowski and J. B. Judkins, Full wave vector Maxwell equation modeling of the selffocusing of ultrashort optical pulses in a nonlinear Kerr medium exhibiting a finite response time, J. Opt; Soc. Am. B, 10(2), 186-198, 1993.

[20] R.-W. Ziolkowski, The incorporation of microscopic material models into FDTD approach for ultrafast optical pulses simulations, IEEE Transactions on Antennas and Propagation, 45(3), 375-391, 1997. 